\title{
New concept of the Axin2 rs2240308 polymorphism and cancer risk: an updated meta-analysis
}

\author{
Y. YU, Y. TAO \\ Department of Epidemiology and Biostatistics, School of Public Health, Nantong University, Nantong, Jiangsu, China \\ ${ }^{*}$ Correspondence: chuminjie@ntu.edu.cn \\ ${ }^{*}$ Contributed equally to this work.
}

Submitted July 25, 2016 / Accepted October 3, 2016

\begin{abstract}
Previous meta-analyses reported that the variant T allele of Axin2 rs2240308 is associated with a decreased cancer risk. However, more recent findings have been inconsistent. Therefore, we carried out an updated meta-analysis to examine whether this polymorphism is still associated with a decreased cancer risk. Twelve articles, including 14 case-control studies (2,215 cases and 2,481 controls), were included in our study. Surprisingly, different from previous meta-analyses, no significant association between Axin 2 rs 2240308 polymorphism and cancer risk was observed (dominant model: OR=0.85; $95 \% \mathrm{CI}=0.68-1.06)$. In further stratified analyses, rs2240308 was significantly associated with a decreased cancer risk only in Asians (dominant model: $\mathrm{OR}=0.76 ; 95 \% \mathrm{CI}=0.66-0.88$ ), while for Caucasians the variant showed no significant association with cancer risk (dominant model: $\mathrm{OR}=1.09 ; 95 \% \mathrm{CI}=0.67-1.76$ ). Moreover, the rs2240308 variant exhibited a significant association with a decreased risk of lung cancer and prostate cancer. These findings provided new evidence that differed from previous meta-analyses; Axin2 rs2240308 may not modify general cancer susceptibility. Similar with previous meta-analyses, our analysis indicated that Axin2 rs2240308 may modify cancer susceptibility in an ethnicity- and/or type-specific manner. These findings indicate that further replication studies with large sample sizes are warranted to re-evaluate the relationship between Axin 2 rs2240308 and cancer risk, especially in Caucasians.
\end{abstract}

Key words: Axin2, rs2240308, polymorphism, cancer, meta-analysis

The continuing global demographic and epidemiologic transitions signal an ever-increasing cancer burden over the next decades, particularly in developing countries, with over 20 million new cancer cases expected annually as early as 2025 [1]. Cancers are considered to be multifactorial diseases, and their occurrences are related to environmental, genetic and lifestyle factors. Remarkably, with the rapid development of genotyping technologies such as genome-wide association studies (GWAS) and next-generation sequencing (NGS), our understanding of the genetic factors that confer cancer risk has substantially broadened.

The Wnt signalling pathway plays a crucial role during embryogenesis, while aberrations in this pathway are implicated in a variety of human cancers. Axin2, a key component of the Wnt signalling pathway, plays an important role in the regulation of cell proliferation, cytometaplasia, migration, apoptosis and other important cellular functions, and it has demonstrated a close relationship with the development of some cancers [2, 3]. Specifically, the relationship between genetic polymorphisms of the Axin2 gene and cancer sensitivity has attracted much interest. The Axin2 rs2240308 polymorphism has been widely implicated in cancer risk; however, the results of studies exploring this association were inconclusive. For example, a previous study reported that a rs2240308 variant genotype significantly increased the risk of colorectal cancer in Mexican individuals [4]. In contrast, the same variant exhibited no significant association of colorectal cancer risk among Iranian people and resulted in an effect value that was opposite relative to the study of Mexican populations [5]. Regardless, an increasing number of studies have given special attention to the association between Axin2 rs2240308 and several common types of cancer such as lung cancer[6-8], prostate cancer $[9,10]$, colorectal cancer $[4,5,8]$, ovarian cancer [11], head and neck cancer [8], breast cancer [12], papillary thyroid carcinoma [13], hepatocellular carcinoma [14] and astrocytoma [15]. Moreover, several studies 
summarized the associations of Axin2 rs2240308 with cancer risk by meta-analyses [16-18]. However, some of these results are controversial. For example, the meta-analysis performed by $\mathrm{Wu}$ et al. did not integrate all the articles that were published at that time (Table 1). Clearly, the association analyses between Axin2 rs2240308 and cancer susceptibility are still necessary. Therefore, we performed an updated meta-analysis using all published data to date to more precisely characterize the association between Axin2 rs2240308 polymorphism and cancer risk.

\section{Materials and methods}

Identification and eligibility of relevant studies. Relevant literature was collected by searching PubMed and Web of Science (the last search update was Aug 31, 2016) using the following keywords: ("Axin2" or "Axis inhibition protein 2") and ("cancer", "carcinoma", "tumor", "tumour", or "neoplasm") and ("polymorphism", "variation", "variant", or "mutation"). Additionally, the references in the retrieved articles were reviewed for possible inclusion. Only publications written in English with available full-text articles were included in this meta-analysis. In this meta-analysis, all studies met the following standards: (1) involved the Axin2 rs2240308 polymorphism and cancer risk; (2) designed as a case-controlled study; and (3) contained available genotype frequency. The chief reasons for exclusion of studies were as follows: (1) not involving the Axin2 gene; (2) not involving rs2240308 polymorphism research; (3) not related to cancer research; and (4) no relevant data reported. Data for meta-analysis were available from 12 articles including 14 case-controlled studies (Figure 1).

Data extraction. Two investigators (Y.Y. and L.L.) independently extracted data and reached consensus on all of the items. The following information, including the first author's name, year of publication, country of origin, ethnicity, type of cancer, numbers of cases and controls, source of controls and genotyping platform, was sought for each article. Ethnicities were categorized as Asians or Caucasians.

Statistical analysis. The risk of cancer associated with Axin2 rs2240308 was estimated for each study using the odds ratio (OR) and its 95\% confidence interval (95\% CI). The between-study heterogeneity was examined with a chisquare-based $Q$ statistical test, and $P \leq 0.05$ was considered as statistically significant. We pooled the results using fixed-effect models when the heterogeneity between studies was absent. Otherwise, a random-effects model was chosen. Subsequently, we evaluated the risks of the heterozygous and variant homozygous genotypes relative to the wild-type homozygous genotype and then assessed the risks of the combined heterozygous as well as variant homozygous genotypes relative to the wild-type homozygous genotype while assuming the dominant effects of the variant allele. In addition, we also assessed the risks of the variant homozygous genotypes relative to the combined variant heterozygous as well as wild-type homozygous genotype while assuming the recessive effects of the variant allele. Additionally, based on ethnicity (divided into Asians and Caucasians), cancer type, source of controls and sample size, we performed stratification analyses. Funnel plots and Begg's test were utilized to evaluate publication bias. All

Table 1. Characteristics of literature included in the meta-analysis

\begin{tabular}{|c|c|c|c|c|c|c|c|c|c|c|c|}
\hline \multirow[b]{2}{*}{ First Author } & \multirow[b]{2}{*}{ Year } & \multirow[b]{2}{*}{ Country } & \multirow[b]{2}{*}{ Ethnicity } & \multirow[b]{2}{*}{ Type of cancer } & \multirow[b]{2}{*}{$\begin{array}{l}\text { Case/ } \\
\text { Control }\end{array}$} & \multirow[b]{2}{*}{ Source of controls } & \multirow[b]{2}{*}{ Platform } & \multirow[b]{2}{*}{$\mathrm{HWE}^{\mathrm{a}}$} & \multicolumn{3}{|c|}{ Included in previous meta-analyses } \\
\hline & & & & & & & & & $\begin{array}{l}\text { Zhong } \\
\text { et al. }{ }^{[16]}\end{array}$ & $\begin{array}{c}\text { Wu } \\
\text { et al. }{ }^{[17]}\end{array}$ & $\begin{array}{c}\text { Gong et } \\
\text { al. }{ }^{[18]}\end{array}$ \\
\hline Rosales-Reynoso & 2016 & Mexican & Caucasian & Colorectal cancer & $201 / 100$ & Population-based & PCR-RFLP & 0.05 & & & \\
\hline Aristizabal-Pachon & 2016 & Brazil & Caucasian & Breast cancer & $102 / 102$ & Hospital-based & PCR-RFLP & 0.00 & & & \\
\hline Kim & 2016 & Korea & Asian & $\mathrm{HCC}^{b}$ & $245 / 483$ & Hospital-based & Golden gate & 0.79 & & & \\
\hline Liu & 2016 & China & Asian & $\mathrm{PTC}^{c}$ & $56 / 50$ & Hospital-based & Sequenom & 0.08 & $\sqrt{ }$ & $\sqrt{ }$ & \\
\hline Liu & 2014 & China & Asian & Lung cancer & $520 / 555$ & Population-based & TaqMan & 0.46 & $\sqrt{ }$ & $\sqrt{ }$ & $\sqrt{ }$ \\
\hline $\mathrm{Ma}$ & 2014 & China & Asian & Prostate cancer & $103 / 100$ & Hospital-based & SNaPshot & 0.15 & $\sqrt{ }$ & $\sqrt{ }$ & $\sqrt{ }$ \\
\hline Mostowska & 2014 & Poland & Caucasian & Ovarian cancer & $258 / 282$ & Hospital-based & $\mathrm{HRM}^{\mathrm{d}}$ & 0.55 & $\sqrt{ }$ & $\sqrt{ }$ & $\sqrt{ }$ \\
\hline Naghibalhossaini & 2012 & Iran & Asian & Colorectal cancer & $110 / 179$ & Not report & PCR-RFLP & 0.10 & $\sqrt{ }$ & & $\sqrt{ }$ \\
\hline Pinarbasi & 2011 & Turkish & Caucasian & Prostate cancer & $84 / 100$ & Hospital-based & PCR-RFLP & 0.88 & $\sqrt{ }$ & $\sqrt{ }$ & $\sqrt{ }$ \\
\hline Gunes & 2010 & Turkish & Caucasian & Astrocytoma & $100 / 100$ & Hospital-based & PCR-RFLP & 0.50 & $\sqrt{ }$ & $\sqrt{ }$ & $\sqrt{ }$ \\
\hline Gunes & 2009 & Turkish & Caucasian & Lung cancer & $100 / 100$ & Not report & PCR-RFLP & 0.50 & $\sqrt{ }$ & $\sqrt{ }$ & $\sqrt{ }$ \\
\hline Kanzaki & 2006 & Japan & Asian & Lung cancer & $160 / 110$ & Population-based & PCR-RFLP & 0.86 & $\sqrt{ }$ & $\sqrt{ }$ & $\sqrt{ }$ \\
\hline Kanzaki & 2006 & Japan & Asian & Colorectal cancer & $113 / 110$ & Population-based & PCR-RFLP & 0.86 & $\sqrt{ }$ & $\sqrt{ }$ & $\sqrt{ }$ \\
\hline Kanzaki & 2006 & Japan & Asian & $\mathrm{HNC}^{\mathrm{e}}$ & $63 / 110$ & Population-based & PCR-RFLP & 0.86 & $\sqrt{ }$ & $\sqrt{ }$ & $\sqrt{ }$ \\
\hline
\end{tabular}

${ }^{a}$ Hardy-Weinberg equilibrium (HWE)

${ }^{\mathrm{b}}$ hepatocellular carcinoma (HCC)

c papillary thyroid carcinoma (PTC)

${ }^{\mathrm{d}}$ high-resolution melting curve analysis (HRM)

${ }^{\mathrm{e}}$ head and neck cancer (HNC) 


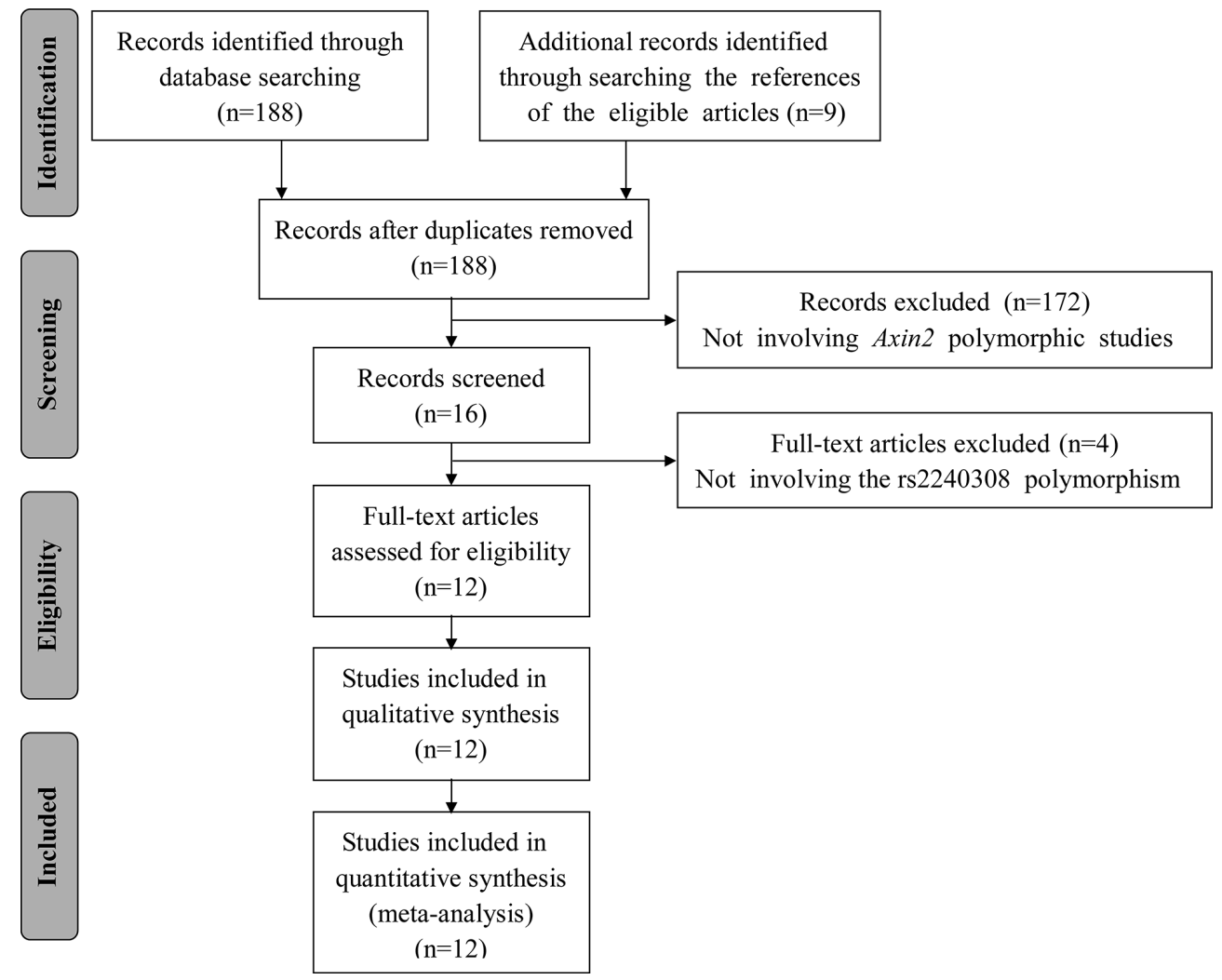

Figure 1. Flow diagram of the study selection process

analyses were performed using the Stata version 12.0 software (Stata Corporation, College Station, TX, USA).

\section{Results}

Characteristics of the published studies. Following the application of strict screening criteria, 12 articles, including 14 case-control studies harbouring a total of 2215 cases and 2481 controls for lung cancer, prostate cancer, colorectal cancer, ovarian cancer, head and neck cancer, breast cancer, papillary thyroid carcinoma, hepatocellular carcinoma and astrocytoma, were ultimately included in the quantitative analysis. The general characteristics of the included studies are listed in Table 1. Among the included studies, 8 were carried out among Asian populations, while 6 studies were carried out in Caucasian populations. The distribution of genotypes among the controls was consistent with Hardy-Weinberg equilibrium (HWE) for 13 studies, with the exception of the study performed by Aristizabal-Pachon et al [12]. There were 3 studies that reported the effects of Axin 2 rs2240308 in lung cancer, 3 studies in colorectal cancer, 2 studies in prostate cancer, and 1 study each for ovarian cancer, astrocytoma, head and neck cancer, breast cancer, papillary thyroid carcinoma, and hepatocellular carcinoma. Genotyping was performed using PCR-RFLP in 9 studies, TaqMan in 1 study, Sequenom in 1 study, $\mathrm{SNaPshot}$ in 1 study, high-resolution melting curve analysis (HRM) in 1 study and Golden gate in 1 study. The distributions of the genotypes and alleles of the Axin2 rs2240308 polymorphism in each individual study are listed in Supplementary Table 1.

Quantitative synthesis. The evaluations of the associations of Axin2 rs2240308 with cancer risks are presented in Table 2. All in all, the variant $\mathrm{T}$ allele exhibited no significant association with cancer risk in the dominant model $(\mathrm{OR}=0.85 ; 95 \%$ $\mathrm{CI}=0.68-1.06, P=0.000$ for the heterogeneity test, $I^{2}=64.4 \%$; Figure 2). The results of other tested models are listed in Table 2 and Figures 3-4.

Next, we evaluated the effect of the rs2240308 polymorphism on cancer risk among the subgroups (Table 2). In the stratified analyses, the rs2240308 SNP had a significant association with decreased cancer risk among Asians (dominant model: $\mathrm{OR}=0.76 ; 95 \% \mathrm{CI}=0.66-0.88 ; P=0.185$ for the heterogeneity test, $\left.I^{2}=30.5 \%\right)$. In addition, the rs 2240308 variant exhibited a significant association with a decreased risk of lung cancer (dominant model: $\mathrm{OR}=0.69 ; 95 \% \mathrm{CI}=0.56-0.85$; $P=0.655$ for the heterogeneity test, $I^{2}=0.0 \%$; Table 2 ) and prostate cancer (dominant model: $\mathrm{OR}=0.62 ; 95 \% \mathrm{CI}=0.41-0.93$; $P=0.078$ for the heterogeneity test, $I^{2}=67.8 \%$; Table 2). Interestingly, the variant $T$ allele of rs 2240308 was significantly associated with decreased cancer risk among studies with 


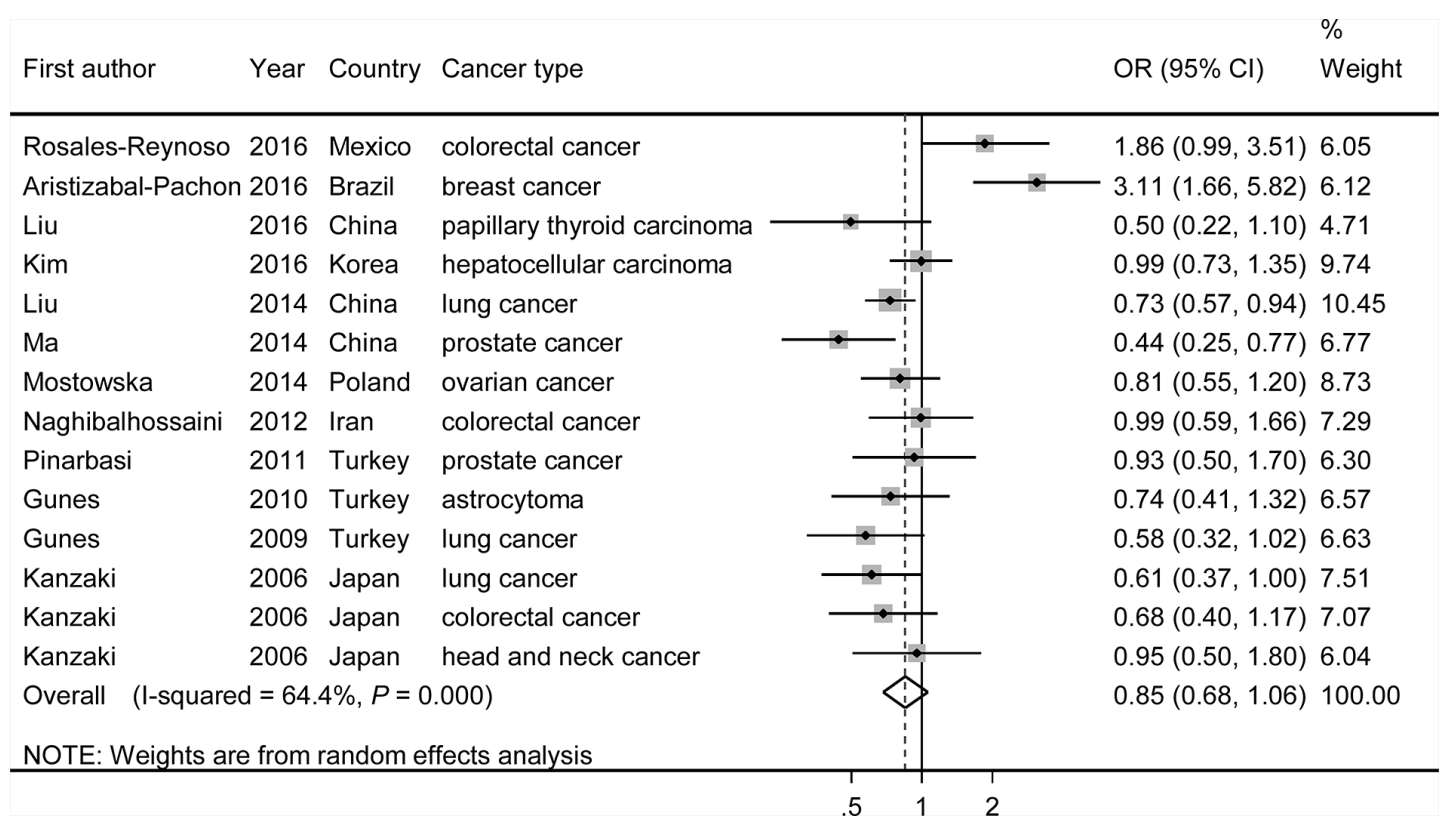

Figure 2. Forest plot of the Axin2 rs2240308 polymorphism and cancer risk in dominant model

population-based controls (dominant model: $\mathrm{OR}=0.78 ; 95 \%$ $\mathrm{CI}=0.65-0.94 ; P=0.061$ for the heterogeneity test, $I^{2}=55.7 \%$; Table 2) and with a sample size $\geq 500$ (dominant model: $\mathrm{OR}=0.82 ; 95 \% \mathrm{CI}=0.69-0.98 ; P=0.325$ for the heterogeneity test, $I^{2}=11.0 \%$; Table 2).

Test of heterogeneity. For rs2240308, significant heterogeneity was observed after the data were pooled (dominant model: $P$ for heterogeneity $=0.000, I^{2}=64.4 \%$; Table 2). When the subjects were stratified on the basis of ethnicity, the heterogeneity disappeared in the Asian groups (dominant model: $P$ for heterogeneity=0.185, $\left.I^{2}=30.5 \%\right)$. Additionally, in stratified analyses based on the source of controls, the heterogeneity disappeared among studies with populationbased controls (dominant model: $P$ for heterogeneity $=0.061$,

Table 2. Summary ORs of the Axin2 rs2240308 polymorphism and cancer risk

\begin{tabular}{|c|c|c|c|c|c|c|c|c|c|c|}
\hline \multirow{2}{*}{ Variables } & \multirow{2}{*}{ Studies } & \multicolumn{3}{|c|}{ CT versus CC } & \multicolumn{3}{|c|}{ TT versus CC } & \multicolumn{3}{|c|}{ Dominant model } \\
\hline & & OR(95\%CI) & $P^{\text {a }}$ & $I^{2}$ & OR $(95 \% \mathrm{CI})$ & $P^{\text {a }}$ & $I^{2}$ & $\mathrm{OR}(95 \% \mathrm{CI})$ & $P^{\text {a }}$ & $I^{2}$ \\
\hline Total & 14 & $0.83(0.68-1.01)$ & 0.018 & $49.6 \%$ & $0.92(0.63-1.34)$ & 0.000 & $69.4 \%$ & $0.85(0.68-1.06)$ & 0.000 & $64.4 \%$ \\
\hline \multicolumn{11}{|l|}{ Ethnicity } \\
\hline Asians & 8 & $0.77(0.66-0.90)$ & 0.173 & $31.9 \%$ & $0.71(0.55-0.92)$ & 0.340 & $11.6 \%$ & $0.76(0.66-0.88)$ & 0.185 & $30.5 \%$ \\
\hline Caucasians & 6 & $1.01(0.69-1.49)$ & 0.024 & $61.4 \%$ & $1.39(0.62-3.15)$ & 0.000 & $83.5 \%$ & $1.09(0.67-1.76)$ & 0.001 & $77.4 \%$ \\
\hline \multicolumn{11}{|l|}{ Cancer type } \\
\hline Lung cancer & 3 & $0.73(0.59-0.91)$ & 0.870 & $0.0 \%$ & $0.52(0.36-0.74)$ & 0.206 & $36.8 \%$ & $0.69(0.56-0.85)$ & 0.655 & $0.0 \%$ \\
\hline Colorectal cancer & 3 & $0.96(0.68-1.34)$ & 0.123 & $52.2 \%$ & $1.36(0.87-2.11)$ & 0.096 & $57.2 \%$ & $1.01(0.74-1.39)$ & 0.060 & $64.4 \%$ \\
\hline Prostate cancer & 2 & $0.54(0.35-0.84)$ & 0.088 & $65.7 \%$ & $1.00(0.54-1.87)$ & 0.509 & $0.0 \%$ & $0.62(0.41-0.93)$ & 0.078 & $67.8 \%$ \\
\hline Others $^{b}$ & 6 & $0.97(0.79-1.18)$ & 0.054 & $54.0 \%$ & $1.16(0.56-2.42)$ & 0.001 & $77.2 \%$ & $0.99(0.66-1.47)$ & 0.003 & $71.8 \%$ \\
\hline \multicolumn{11}{|l|}{ Source of controls } \\
\hline Population-based & 5 & $0.81(0.66-0.98)$ & 0.237 & $27.6 \%$ & $0.82(0.43-1.58)$ & 0.004 & $73.9 \%$ & $0.78(0.65-0.94)$ & 0.061 & $55.7 \%$ \\
\hline Hospital-based & 7 & $0.83(0.57-1.19)$ & 0.004 & $68.6 \%$ & $1.12(0.60-2.07)$ & 0.001 & $73.2 \%$ & $0.88(0.59-1.30)$ & 0.000 & $75.6 \%$ \\
\hline Not report & 2 & $0.79(0.53-1.18)$ & 0.354 & $0.0 \%$ & $0.75(0.43-1.33)$ & 0.051 & $73.7 \%$ & $0.78(0.53-1.14)$ & 0.167 & $47.7 \%$ \\
\hline \multicolumn{11}{|l|}{ Sample size } \\
\hline$\geq 500$ & 3 & $0.85(0.71-1.02)$ & 0.387 & $0.0 \%$ & $0.72(0.54-0.95)$ & 0.666 & $0.0 \%$ & $0.82(0.69-0.98)$ & 0.325 & $11.0 \%$ \\
\hline$<500$ & 11 & $0.82(0.62-1.09)$ & 0.008 & $58.0 \%$ & $1.00(0.57-1.75)$ & 0.000 & $74.4 \%$ & $0.86(0.62-1.19)$ & 0.000 & $70.8 \%$ \\
\hline
\end{tabular}

${ }^{a}$ Random-effects model was used when $P$ value for heterogeneity test $<0.05$; otherwise, fixed-effect model was used

${ }^{\mathrm{b}}$ others include ovarian cancer, head and neck cancer, breast cancer, papillary thyroid carcinoma, hepatocellular carcinoma and astrocytoma 


\begin{tabular}{|c|c|c|c|c|c|c|}
\hline First author & Year & Country & Cancer type & & OR $(95 \% \mathrm{Cl})$ & $\begin{array}{l}\% \\
\text { Weight }\end{array}$ \\
\hline Rosales-Reynoso & 2016 & Mexico & colorectal cancer & 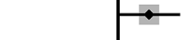 & $1.81(0.99,3.31)$ & 8.73 \\
\hline Aristizabal-Pachor & 2016 & Brazil & breast cancer & & $10.15(2.95,34.96)$ & 4.07 \\
\hline Kim & 2016 & Korea & hepatocellular carcinoma & & $0.86(0.49,1.54)$ & 8.98 \\
\hline Liu & 2016 & China & papillary thyroid carcinoma & & $0.45(0.08,2.58)$ & 2.40 \\
\hline Liu & 2014 & China & lung cancer & $\rightarrow$ & $0.72(0.49,1.08)$ & 10.93 \\
\hline $\mathrm{Ma}$ & 2014 & China & prostate cancer & & $1.21(0.48,3.06)$ & 5.86 \\
\hline Mostowska & 2014 & Poland & ovarian cancer & & $0.84(0.55,1.29)$ & 10.60 \\
\hline Naghibalhossaini & 2012 & Iran & colorectal cancer & & $1.23(0.64,2.34)$ & 8.27 \\
\hline Pinarbasi & 2011 & Turkey & prostate cancer & o & $1.33(0.65,2.74)$ & 7.54 \\
\hline Gunes & 2010 & Turkey & astrocytoma & & $1.00(0.47,2.13)$ & 7.23 \\
\hline Gunes & 2009 & Turkey & lung cancer & 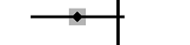 & $0.46(0.19,1.12)$ & 6.07 \\
\hline Kanzaki & 2006 & Japan & lung cancer & $\longrightarrow$ & $0.33(0.13,0.81)$ & 6.09 \\
\hline Kanzaki & 2006 & Japan & colorectal cancer & & $0.96(0.44,2.07)$ & 7.11 \\
\hline Kanzaki & 2006 & Japan & head and neck cancer & & $1.04(0.43,2.55)$ & 6.12 \\
\hline \multicolumn{4}{|c|}{ Overall $(I-$ squared $=58.5 \%, P=0.003)$} & $\Phi$ & $1.00(0.74,1.34)$ & 100.00 \\
\hline NOTE: Weights ar & e from & random $\mathrm{e}$ & effects analysis & & & \\
\hline
\end{tabular}

Figure 3. Forest plot of the Axin2 rs2240308 polymorphism and cancer risk in recessive model

$\left.I^{2}=55.7 \%\right)$. Moreover, when the studies were stratified on the basis of cancer type, the heterogeneity disappeared for lung cancer (dominant model: $P$ for heterogeneity $=0.655$, $I^{2}=0.0 \%$ ), colorectal cancer (dominant model: $P$ for heterogeneity $\left.=0.060, I^{2}=64.4 \%\right)$ and prostate cancer (dominant model: $P$ for heterogeneity $=0.078, I^{2}=67.8 \%$ ). Furthermore, the heterogeneity also disappeared among studies with a sample size $\geq 500$ (dominant model: $P$ for heterogeneity $=0.325, I^{2}=11.0 \%$ ).

Sensitivity analysis. To test the stability of the rs 2240308 results, we conducted sensitivity analyses by sequentially removing each eligible study (Supplementary Table 2). The study by Aristizabal-Pachon et al. [12] that focused on breast cancer was the major contributor of heterogeneity in the

Table 2. Summary ORs of the Axin2 $\mathrm{rs} 2240308$ polymorphism and cancer risk (Recessive model and $\mathrm{T}$ versus $\mathrm{C}$ model)

\begin{tabular}{|c|c|c|c|c|c|c|c|}
\hline \multirow{2}{*}{ Variables } & \multirow{2}{*}{ Studies } & \multicolumn{3}{|c|}{ Recessive model } & \multicolumn{3}{|c|}{$\mathrm{T}$ versus $\mathrm{C}$} \\
\hline & & $\mathrm{OR}(95 \% \mathrm{CI})$ & $P^{\text {a }}$ & $I^{2}$ & OR $(95 \% C I)$ & $P^{\text {a }}$ & $I^{2}$ \\
\hline Total & 14 & $1.00(0.74-1.34)$ & 0.003 & $58.5 \%$ & $0.92(0.77-1.10)$ & 0.000 & $72.9 \%$ \\
\hline \multicolumn{8}{|l|}{ Ethnicity } \\
\hline Asians & 8 & $0.82(0.64-1.04)$ & 0.383 & $6.1 \%$ & $0.82(0.74-0.92)$ & 0.240 & $23.8 \%$ \\
\hline Caucasians & 6 & $1.32(0.73-2.37)$ & 0.001 & $75.9 \%$ & $1.11(0.77-1.60)$ & 0.000 & $83.7 \%$ \\
\hline \multicolumn{8}{|l|}{ Cancer type } \\
\hline Lung cancer & 3 & $0.61(0.43-0.85)$ & 0.230 & $32.0 \%$ & $0.73(0.63-0.85)$ & 0.398 & $0.0 \%$ \\
\hline Colorectal cancer & 3 & $1.36(0.93-1.99)$ & 0.413 & $0.0 \%$ & $1.10(0.90-1.35)$ & 0.071 & $62.2 \%$ \\
\hline Prostate cancer & 2 & $1.28(0.73-2.27)$ & 0.872 & $0.0 \%$ & $0.83(0.62-1.12)$ & 0.099 & $63.2 \%$ \\
\hline Others $^{b}$ & 6 & $1.16(0.66-2.06)$ & 0.008 & $67.8 \%$ & $1.03(0.74-1.43)$ & 0.000 & $79.7 \%$ \\
\hline \multicolumn{8}{|l|}{ Source of controls } \\
\hline Population-based & 5 & $0.88(0.53-1.45)$ & 0.023 & $64.6 \%$ & $0.89(0.67-1.17)$ & 0.008 & $71.2 \%$ \\
\hline Hospital-based & 7 & $1.20(0.74-1.94)$ & 0.013 & $62.7 \%$ & $0.98(0.72-1.32)$ & 0.000 & $79.0 \%$ \\
\hline Not report & 2 & $0.86(0.51-1.45)$ & 0.079 & $67.5 \%$ & $0.86(0.66-1.11)$ & 0.062 & $71.3 \%$ \\
\hline \multicolumn{8}{|l|}{ Sample size } \\
\hline$\geq 500$ & 3 & $0.79(0.61-1.03)$ & 0.833 & $0.0 \%$ & $0.85(0.75-0.97)$ & 0.398 & $0.0 \%$ \\
\hline$<500$ & 11 & $1.09(0.71-1.67)$ & 0.002 & $63.6 \%$ & $0.94(0.73-1.21)$ & 0.000 & $77.5 \%$ \\
\hline
\end{tabular}

${ }^{a}$ Random-effects model was used when $P$ value for heterogeneity test $<0.05$; otherwise, fixed-effect model was used

${ }^{\mathrm{b}}$ others include ovarian cancer, head and neck cancer, breast cancer, papillary thyroid carcinoma, hepatocellular carcinoma and astrocytoma 


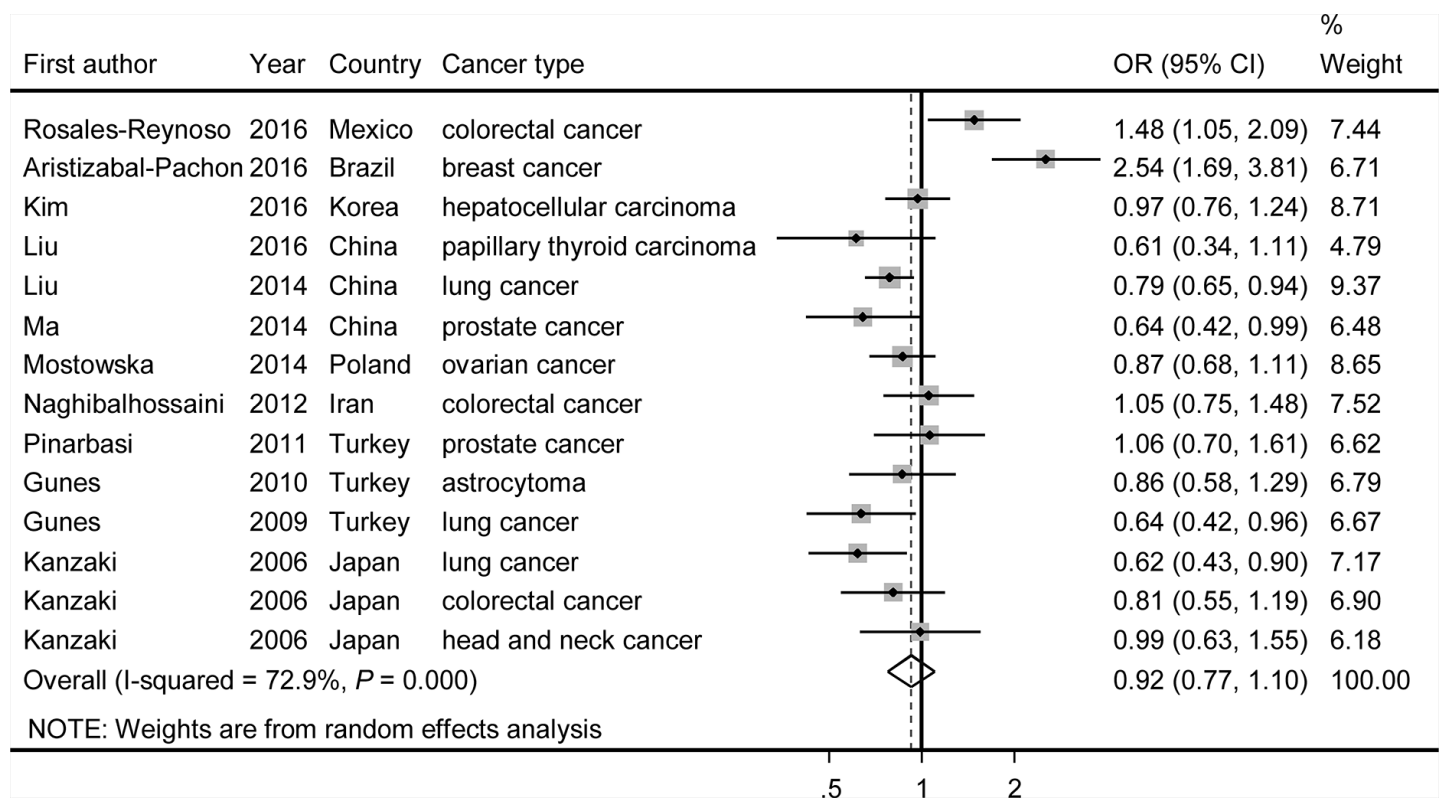

Figure 4. Forest plot of the Axin2 rs2240308 polymorphism and cancer risk in allele (T vs C) model

dominant model $\left(I^{2}=64.4 \%, P\right.$ for heterogeneity $\left.=0.000\right)$. After the removal of this study, the heterogeneity was significantly reduced $\left(I^{2}=36.3 \%, P\right.$ for heterogeneity $\left.=0.093\right)$. As expected, similar results were observed in other genetic models (i.e., CT versus $\mathrm{CC}$ and TT versus CC), indicating that the study by Aristizabal-Pachon et al. [12] emphasizing breast cancer markedly changed the pooled OR.

Publication bias. We utilized funnel plots and Begg's test to evaluate potential publication biases of the studied literature. The shapes of the funnel plots were symmetrical (Figure 5). Moreover, a Begg's test provided further statistical evidence for the absence of publication bias (dominant model: $P=0.70$ ).

\section{Discussion}

Previous meta-analyses reported that the variant $\mathrm{T}$ allele of Axin2 rs2240308 was associated with a decreased cancer risk. However, more recent findings have been inconsistent. Therefore, we performed an updated meta-analysis to examine whether this polymorphism was associated with a decreased cancer risk. In this study, we performed a meta-analysis by pooling 12 articles, including 14 case-control studies $(2,215$ cases and 2,481 controls), and demonstrated no significant association between Axin2 rs2240308 polymorphism and cancer risk. However, similar with previous meta-analysis, in stratified analyses, rs 2240308 was associated with a decreased cancer risk in Asian populations.

The SNP rs2240308 at $17 \mathrm{q} 24.1$ is a missense mutation located at exon 1 of Axin2. Axin2 is an important tumour suppressor, and methylation and mutation of the Axin2 gene could cause abnormal expression of Axin2, resulting in tumourigenesis [2]. In addition, Axin2 may control the level of $\beta$-catenin in the cytoplasm by promoting $\beta$-catenin degradation, thereby functioning as a negative regulator of the Wnt signalling pathway $[19,20]$. Given the importance of the Wnt signalling pathway for adult stem cell biology, it is not surprising that Wnt signalling pathway mutations are frequently observed in cancers, most notably of tissues that normally depend on Wnt for self-renewal or repair[19].

We further performed functional annotation for the Axin2 rs2240308 variant based on publicly available datasets or tools. PolyPhen-2 (http://genetics.bwh.harvard. edu/pph2/) predicted that amino acid substitution resulting from rs2240308 may be damaging and affect the normal function of the Axin 2 protein. We then evaluated whether the rs2240308 variant modulated mRNA expression levels through transcriptional mechanisms. Based on the Encyclopedia of DNA Elements (ENCODE) DNase I hypersensitive site (DHS) sequencing data set, we found that rs 2240308 is within open chromatin regions associated with gene regulatory elements, indicating that rs2240308 may affect transcription factor binding. Furthermore, ChIP-Seq data from the ENCODE project showed that rs 2240308 was located in a region that may affect the binding of numerous transcription factors, including MYC, CTBP2 and MBD4. The MYC transcription factor acts as an oncogene that was overexpressed in lung cancer cells and was associated with lung cancer metastasis $[21,22]$. CTBP2 plays a significant role in tumour initiation, progression and response to therapy, which could promote prostate cancer cell proliferation through c-Myc signalling [23]. MBD4 protein functions as 

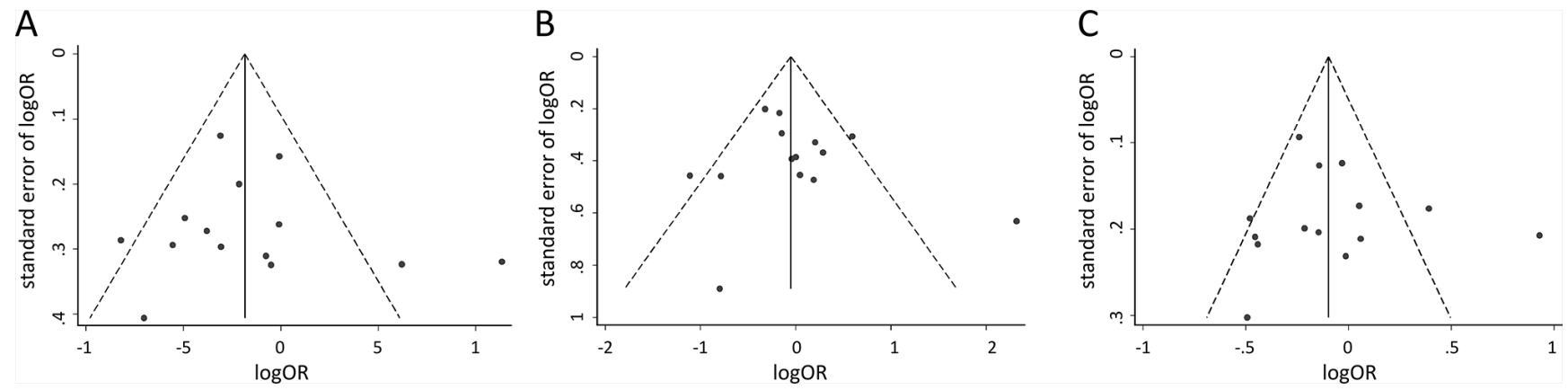

Figure 5. A) funnel plot of the Axin2 rs2240308 polymorphism and cancer risk in dominant model, B) funnel plot of the Axin2 rs2240308 polymorphism and cancer risk in recessive model, C) funnel plot of the Axin2 rs2240308 polymorphism and cancer risk in allele (T vs C) model

a DNA repair enzyme and plays an important role in maintaining genome integrity and carcinogenesis. In addition, MBD4 Glu346Lys polymorphism is associated with cervical cancer susceptibility in Chinese populations [24]. It is plausible that variation in the Axin2 rs2240308 may result in the aberrant activities of certain transcription factors. In turn, those factors may regulate the expression of the same target genes nearby or throughout the genome, hence activating crucial signalling pathways that are involved in carcinogenesis. However, these results are preliminary and require further experimental investigation.

Different from previous meta-analyses, in our study no overall significant association existed with Axin2 rs2240308 polymorphism and any cancer type. However, in subsequent analyses stratified by ethnicity, rs2240308 was associated with a decreased cancer risk in Asians, while no significant association was observed in Caucasians. There are several reasons for the inconsistent results. First, the difference may be due to genetic heterogeneity between different ethnicities. Second, the difference may owe to the utilization of different genotyping methods, which included PCR-RFLP, Sequenom, TaqMan Real-Time PCR, etc. Additionally, different types of cancer involve random errors, which may also potentially account for the differences in findings between Asian and Caucasian populations.

Nevertheless, further studies with large sample sizes are warranted to evaluate the relationship between rs2240308 and cancer risk, especially in Caucasians.

The strength of this meta-analysis is that we systemically reviewed the relationships between Axin2 rs2240308 and tumour susceptibility using all published data up to the present moment (the last search update was Aug 31, 2016). In contrast, it seems that the previous meta-analyses performed by Wu et al. and Gong et al. did not integrate all articles that were published at that time, which may have affected the accuracy of the results. Therefore, different from previous meta-analyses, our study provided precise evidence for the first time that Axin2 rs2240308 may not modify general cancer susceptibility. Similar findings to previous meta-analyses were that Axin2 rs2240308 may modify cancer susceptibility in an ethnicity- and/or type-specific way, which may be valuable for the research of tumour pathogenesis mechanisms and identification of potential diagnostic targets. In addition, compared with previous meta-analyses, in the stratified analyses we have reported for the first time that a variant $\mathrm{T}$ allele of rs2240308 was significantly associated with a decreased cancer risk among studies with relatively large sample sizes $(\geq 500$ ), indicating the important role of sample size in study design. Moreover, the well-designed functional annotation that further verified our findings is another advantage of this study. However, there are also some limitations that must be addressed. First, significant heterogeneity between studies was observed. Among the 12 published articles containing 14 case-control studies included in our meta-analysis, some of the studies were population-based, while others were hospitalbased. Second, in some studies, detailed information (e.g., age, gender, smoking status, and alcohol consumption) was not provided, which limited further stratification analyses. Additionally, if we had been able to acquire more detailed information, we would have achieved more precise estimations by adjusting for other potential covariates. Third, we did not consider the possibility that different cancers have different incidence/prevalence rates in different populations, so our conclusions should be interpreted cautiously. Fourth, for the study performed by Kanzaki et al., they used the same controls for different cancer types, which may affect the accuracy of the results of their study. Lastly, for the studies performed in Mexico and Brazil, the authors did not clarify whether the research subjects were native Mexicans or Brazilians. Further, we could not exclude the possibility that study subjects from Mexico and Brazil may not be pure populations, as well as there may be some cases that are not Caucasian; therefore, our conclusions should also be interpreted cautiously.

\section{Conclusions}

This updated meta-analysis provided new evidence that differed from previous meta-analyses. Specifically, Axin2 
rs2240308 may not modify general cancer susceptibility. Similar with previous meta-analyses, our analysis indicated that Axin 2 rs2240308 may modify cancer susceptibility in an ethnicity- and/or type-specific manner. In addition to functional evaluations, future studies incorporating subjects from different ethnic backgrounds combined with re-sequencing of the marked region of Axin2 are warranted.

Supplementary information is available in the online version of the paper.

Acknowledgments: This work was funded by the National Natural Science Foundation of China (81502876), the Natural Science Research of Jiangsu Higher Education Institutions (15KJB330006), the Science and Technology Program of Nantong City (MS22015088) and the Doctoral Scientific Research Foundation of Nantong University (14R16). The funding sources had no role to play in the study design, the collection and interpretation of the data, writing of the report, or decision to submit this paper for publication.

\section{References}

[1] FERLAY J, SOERJOMATARAM I, DIKSHIT R, ESER $S$, MATHERS $C$ et al. Cancer incidence and mortality worldwide: sources, methods and major patterns in GLOBOCAN 2012. Int J Cancer 2015; 136: E359-386. https://doi. org/10.1002/ijc.29210

[2] LI S, WANG C, LIU X, HUA S, LIU X. The roles of AXIN2 in tumorigenesis and epigenetic regulation. Fam Cancer 2015; 14: 325-331. https://doi.org/10.1007/s10689-014-9775-7

[3] SONG X, WANG S. LI L. New insights into the regulation of Axin function in canonical Wnt signaling pathway. Protein Cell 2014; 5: 186-193. https://doi.org/10.1007/s13238-014$\underline{0019-2}$

[4] ROSALES-REYNOSO MA, ARREDONDO-VALDEZ AR, WENCE-CHAVEZ LI, BARROS-NUNEZ P, GALLEGOSARREOLA MP et al. AXIN2 Polymorphisms and Their Association with Colorectal Cancer in Mexican Patients. Genet Test Mol Biomarkers 2016; 20: 438-444. https://doi. org/10.1089/gtmb.2016.0026

[5] NAGHIBALHOSSAINI F, ZAMANI M, MOKARRAM P, KHALILI I, RASTI $M$ et al. Epigenetic and genetic analysis of WNT signaling pathway in sporadic colorectal cancer patients from Iran. Mol Biol Rep 2012; 39: 6171-6178. https://doi. org/10.1007/s11033-011-1434-6

[6] LIU D, LI L, YANG Y, LIU W, WU J. The Axin2 rs2240308 polymorphism and susceptibility to lung cancer in a Chinese population. Tumour Biol 2014; 35: 10987-10991. https://doi. org/10.1007/s13277-014-2399-6

[7] GUNES EG, PINARBASI E, PINARBASI H, SILIG Y. Strong association between lung cancer and the AXIN2 polymorphism. Mol Med Rep 2009; 2: 1029-1035.

[8] KANZAKI H, OUCHIDA M, HANAFUSA H, YANO M, SUZUKI H et al. Single nucleotide polymorphism of the AXIN2 gene is preferentially associated with human lung cancer risk in a Japanese population. Int J Mol Med 2006; 18: 279-284. https://doi.org/10.3892/ijmm.18.2.279
[9] MA C, LIU C, HUANG P, KAKU H, CHEN J et al. Significant association between the Axin2 rs2240308 single nucleotide polymorphism and the incidence of prostate cancer. Oncol Lett 2014; 8: 789-794. https://doi.org/10.3892/ $\underline{\text { ol.2014.2177 }}$

[10] PINARBASI E, GUNES EG, PINARBASI H, DONMEZ G, SILIG Y. AXIN2 polymorphism and its association with prostate cancer in a Turkish population. Med Oncol 2011; 28: 1373-1378. https://doi.org/10.1007/s12032-010-9588-y

[11] MOSTOWSKA A, PAWLIK P, SAJDAK S, MARKOWSKA J, PAWALOWSKA $M$ et al. An analysis of polymorphisms within the Wnt signaling pathway in relation to ovarian cancer risk in a Polish population. Mol Diagn Ther 2014; 18: 85-91. https://doi.org/10.1007/s40291-013-0059-y

[12] ARISTIZABAL-PACHON AF, CARVALHO TI, CARRARA HH, ANDRADE J, TAKAHASHI CS. AXIN2 Polymorphisms, the beta-Catenin Destruction Complex Expression Profile and Breast Cancer Susceptibility. Asian Pac J Cancer Prev 2015; 16: 7277-7284. https://doi.org/10.7314/ APJCP.2015.16.16.7277

[13] LIU X, LI S, LIN X, YAN K, ZHAO L et al. AXIN2 is Associated With Papillary Thyroid Carcinoma. Iran Red Crescent Med J 2016; 18: e20960. https://doi.org/10.5812/ ircmj.20960

[14] KIM SS, CHO HJ, LEE HY, PARK JH, NOH CK et al. Genetic polymorphisms in the Wnt/beta-catenin pathway genes as predictors of tumor development and survival in patients with hepatitis B virus-associated hepatocellular carcinoma. Clin Biochem 2016; 49: 792-801. https://doi.org/10.1016/j. clinbiochem.2016.01.025

[15] GUNES EG, PINARBASI E, PINARBASI H. AXIN2 polymorphism and its association with astrocytoma in a Turkish population. Mol Med Rep 2010; 3: 705-709.

[16] ZHONG A, PAN X, SHI M AND XU H. -148 C/T polymorphism of Axin2 contributes to a decreased risk of cancer: evidence from a meta-analysis. Onco Targets Ther 2015; 8: 1957-1966. https://doi.org/10.2147/OTT.S86738

[17] WU Z, SUN Y, TANG S, LIU C, ZHU S et al. AXIN2 rs2240308 polymorphism contributes to increased cancer risk: evidence based on a meta-analysis. Cancer Cell Int 2015; 15: 68. https:// doi.org/10.1186/s12935-015-0219-8

[18] GONG J, JIANG Y, HAO N, ZHU B, LI Y. Quantitative assessment of the association between AXIN2 rs2240308 polymorphism and cancer risk. Sci Rep 2015; 5: 10111. https:// doi.org/10.1038/srep 10111

[19] CLEVERS H, NUSSE R. Wnt/beta-catenin signaling and disease. Cell 2012; 149: 1192-1205. https://doi.org/10.1016/j. cell.2012.05.012

[20] BEHRENS J, JERCHOW BA, WURTELE M, GRIMM J, ASBRAND $\mathrm{C}$ et al. Functional interaction of an axin homolog, conductin, with beta-catenin, APC, and GSK3beta. Science 1998; 280: 596-599. https://doi.org/10.1126/ science. 280.5363 .596

[21] RAPP UR, KORN C, CETECI F, KARREMAN C, LUETKENHAUS K et al. MYC is a metastasis gene for non-small-cell lung cancer. PLoS One 2009; 4: e6029. https://doi.org/10.1371/ journal.pone.0006029 
[22] ZAJAC-KAYE M. Myc oncogene: a key component in cell cycle regulation and its implication for lung cancer. Lung Cancer 2001; 34 Suppl 2: S43-46. https://doi.org/10.1016/ $\underline{\text { S0169-5002(01)00343-9 }}$

[23] ZHANG C, GAO C, XU Y, ZHANG Z. CtBP2 could promote prostate cancer cell proliferation through c-Myc signaling. Gene 2014; 546: 73-79. https://doi.org/10.1016/j.gene.2014.05.032
[24] XIONG XD, LUO XP, LIU X, JING X, ZENG LQ et al. The MBD4 Glu346Lys polymorphism is associated with the risk of cervical cancer in a Chinese population. Int J Gynecol Cancer 2012; 22: 1552-1556. https://doi.org/10.1097/ igc.0b013e $31826 \mathrm{e} 22 \mathrm{e} 4$ 